\title{
Interactive Real-Time Simulation and Auralization for Modifiable Rooms \\ by
}

Sönke Pelzer, Lukas Aspöck, Dirk Schröder and Michael Vorländer

Reprinted from

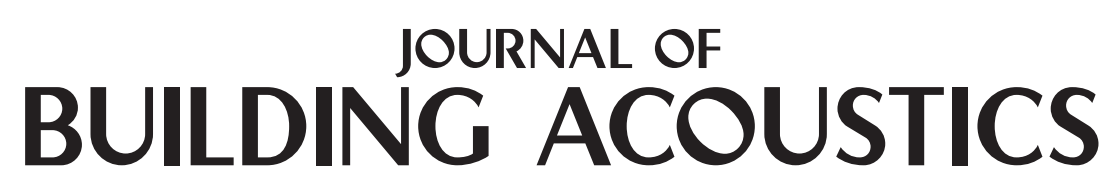

Volume $21 \cdot$ Number $1 \cdot 2014$ 


\title{
Interactive Real-Time Simulation and Auralization for Modifiable Rooms
}

\author{
Sönke Pelzer ${ }^{1}$, Lukas Aspöck ${ }^{1}$, Dirk Schröder ${ }^{2}$ \\ and Michael Vorländer ${ }^{1}$ \\ ${ }^{1}$ Institute of Technical Acoustics, RWTH Aachen University, \\ Kopernikusstr. 5, 52074 Aachen, Germany, \\ spe@akustik.rwth-aachen.de,las@akustik.rwth-aachen.de, \\ mvo@akustik.rwth-aachen.de \\ ${ }^{2}$ Audiovisual Communications Laboratory (LCAV), \\ Ecole Polytechnique Fédérale de Lausanne (EPFL), \\ Station 141015 Lausanne, Switzerland, \\ dirk.schroeder@epfl.ch
}

\begin{abstract}
To study the effects of any changes to a room or setting on the room acoustics, a framework was developed that enables immediate acoustic and visual feedback to the user. This is achieved by running interactive room acoustics simulations and auralizations in real-time. Physically based binaural room impulse responses (BRIRs) are calculated using the image source method and ray tracing and are divided into direct sound, early reflections and late reverberation. Any part of the BRIR is updated as quickly as possible depending on the user's interaction with the scene. This includes changes to sources and receivers (positions/orientations/directivities/HRTF), to surface materials and to the room geometry itself. Using streaming low-latency convolution, an immediate feedback is provided to the user. A parallelization concept features multi-threading and networked PC-clusters, so that the workload can be effectively distributed, offering a scalability to simulate small to huge scenes, depending on the available computation power. For convenient scene design and interaction, a plug-in for Trimble SketchUp was developed that enables real-time room acoustics and room acoustics parameter visualization to this easy-to-use CAD modeling tool.
\end{abstract}

\section{INTRODUCTION}

In the planning and design process of many buildings and spaces, the architect mainly focuses on the visual appearance, neglecting the consequences of his choice on shape and materials on the acoustics. To avoid flaws in the visual domain, it is common practice to perform a so called rendering of a building or scenery, which allows to have a photorealistic view of the construction. This technique has an acoustical counterpart which is called 
auralization. Unfortunately, in contrast to commonly accepted visual renderings this method is not yet broadly established to anticipate the acoustics in the same way.

One reason for this is that the available auralization programs are not well integrated into the common workflow of architectural design and their tools. Auralization programs are usually expensive and difficult to operate compared to visual renderers. Also the production and evaluation of results is not possible without deeper knowledge.

To provide a steep learning curve in acoustics for non-expert users, it is important to have a short actio-reactio circle. This means that the consequences of modifications by the user to the room size, shape and surface materials should be immediately visible and audible. Of course, this immediate feedback would be appreciated by professional users, too.

This immediate feedback should include objective measures, such as the typical room acoustics parameters reverberation time, clarity, strength, etc., as well as the live listening into the scene by means of headphones or loudspeakers.

\section{STATE OF THE ART}

With various room acoustics simulation tools being available today ${ }^{1}$, an acoustic consultant is able to provide predictions for different room acoustic situations. The accuracy of different room acoustic simulation software have been evaluated and compared to measurements ${ }^{2-5}$. Fast computer systems are the basis for generating room impulse responses in a short period of time, in optimized systems results can be provided even in real-time. Next to the application of room acoustics simulation for the designing process of a room, the reduced calculation times led to an application of room acoustics simulation and auralization in Virtual Reality ${ }^{6}$ and can also be integrated in computer games ${ }^{7}$. Systems including room acoustics simulation are usually based on geometrical acoustics, the ray-based simulation techniques guarantee sufficient results in the mid and high frequency bands. Because the Schroeder Frequency ${ }^{10}$ is lower than $50 \mathrm{~Hz}$ for typical room scenarios such as concert halls, the missing consideration of wave-based effects does not influence the outcomes of the simulation significantly. Most of the today's room acoustic simulation software is based on hybrid simulation models (e.g. ODEON, CATT or EASE), combining the image sources model and different types of ray-tracing algorithms. Up to a level, the results of these simulations are satisfactory for the user and the generated room impulse responses can be used for predictions regarding design decisions of rooms. Although in the named applications, an auralization of the room acoustics is possible, these auralizations are restricted to static scenes and do not allow to modifiy all components of the scenery. While in some cases alterations of receiver characteristics (position, orientation, directivity) may be possible, the geometry, wall materials and often source positions cannot be modified during an auralization. As an example the recent CATT-acoustics module ${ }^{8}$ should be mentioned here, allowing for an architectural walkthrough with freely movable receiver. However, the functionality expects pre-simulated impulse responses which are then interpolated.

\section{MOTIVATION}

After recent advances in simulation methods and computer hardware, it is possible to provide a fully interactive CAD modeling tool that simulations correctly on the fly in the 
background, so that no restrictions on interaction or scene design need to be considered. Only without any precalculations, this high level of interactivity can be reached.

Instead of developing another 3D modeler inside an acoustic simulation software, the authors decided to include a fully operable real-time simulation model inside an existing CAD modeler. Therefore a plug-in was developed for Trimble SketchUp a (formerly Google SketchUp), which communicates using a TCP/IP network connection with a simulation backbone.

This concept allows the user (e.g. an architect) to model the space while wearing headphones and any action has immediate effect on the perceived sound scape. Also room acoustics parameters are visualized and updated in real-time. This tool enables an intuitive way to keep attention to the acoustics of any modeled space, being a classroom or an airport terminal, avoiding mistakes and speeding up the workflow.

\section{VIRTUAL ACOUSTICS}

For real-time room acoustics acoustic rendering, the software framework Raven ${ }^{9}$ was developed at the Institute of Technical Acoustics in Aachen. This software is applied e.g. for the immersive 3-D sound reproduction in virtual environments, such as the aixCAVE ${ }^{\mathrm{b}}$. The Raven-Libraries are also the core of the backbone for the SketchUp-Plug-in.

\subsection{Simulation components}

In geometrical acoustics, the simulation of room impulse responses is rarely performed based on only one simulation model. Commonly a hybrid approach combining the Image Sources model ${ }^{11}$ and a Ray Tracing ${ }^{12}$ model is applied to generate accurate simulation results. A typical room impulse response consists of the direct sound, early reflections and a reverberation tail (see Figure 1).

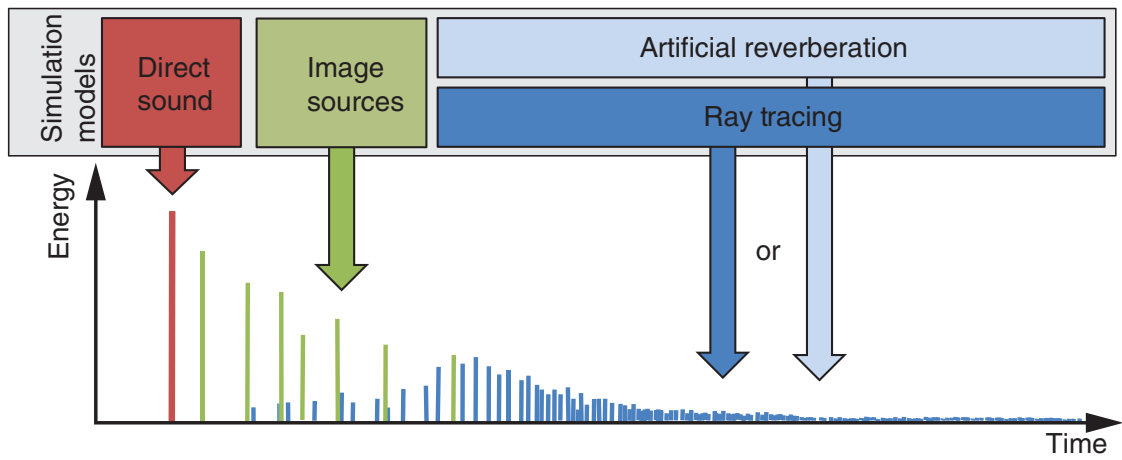

Figure 1. Simulation components and their contributions to the room impulse response.

\footnotetext{
${ }^{\mathrm{a} h t t p: / / w w w . s k e t c h u p . c o m / ~}$

baixCAVE at RWTH Aachen University: http://www.rz.rwth-aachen.de/li/c/tos
} 
The direct sound does not require a particular calculation model; therefore the path between sound source and receiver is checked for polygons in the line of sight. According to source and receiver characteristics, the distance and the air absorption, a direct sound impulse is set into the impulse response. The early reflections, usually up to an order of two to three, are efficiently calculated by the Image Source model, which only accounts for specular wall reflections. All other incoming reflections are determined by the ray tracing algorithm, generating a diffuse reverberation tail, which mostly covers the late part of the room impulse response, but also early diffuse reflections. In comparison, the ray tracing requires much more calculation time compared to the calculation of direct sound and early reflections. This is due to a large number of required rays, describing the sound paths for the high number of reflections in the late part of the reverberation tail. Additionally, because of frequency dependent scattering and absorption coefficients, the ray tracing has to be performed for each frequency band. Ray tracing is nevertheless a suitable simulation model, because the reverberation of a room is only varying slightly and thus requires only low update rates, in contrast to direct sound and early reflections. However, in case of low calculation power of a real-time system, an alternative method to model the reverberation tail of an impulse response is the usage of statistical artificial reverberation, based on the reverberation time determined by the Eyring reverberation time formula. This artificial reverberation is solely dependent on the room size and the absorption coefficients and therefore can be calculated in a rather short period of time.

The separation of the simulation models makes it possible to work with independent update rates and calculation priorities, allowing quick updates for direct sound and image sources without violating real-time constraints in highly interactive situations.

\subsection{Room model}

In geometrical acoustics, the most frequently used operation is the search for an intersection point of a line with the polygons of the room or scene. Therefore a crucial part of the implementation is the spatial data structure which organizes the polygons to speed up these intersections. Progress in this field was mainly driven by advances in the computer graphics domain. Early 3D applications introduced the Binary Space Partitioning (BSP) technique which allows typically the fastest intersection tests. But for any changes in the geometry or moving objects, the underlying BSP tree has to be updated, which is a costly operation. Consequently in modern interactive and dynamic applications other techniques are prevailing which are object-oriented instead of the space-oriented BSPs. Two object-oriented representatives are the Bounding Volume Hierarchy $(\mathrm{BVH}$, fast on moving object collision tests) and the Spatial Hashing ( $\mathrm{SH}$, fast on geometry modifications).

The question now is which of these techniques should be used if the scene is continuously modified by the CAD operator ( $\mathrm{SH}$ advantageous) but also very fast intersections are necessary for the real-time room acoustics (BSP advantageous). A simple answer would be: both of them, as proposed in Figure 2. The SH map is 


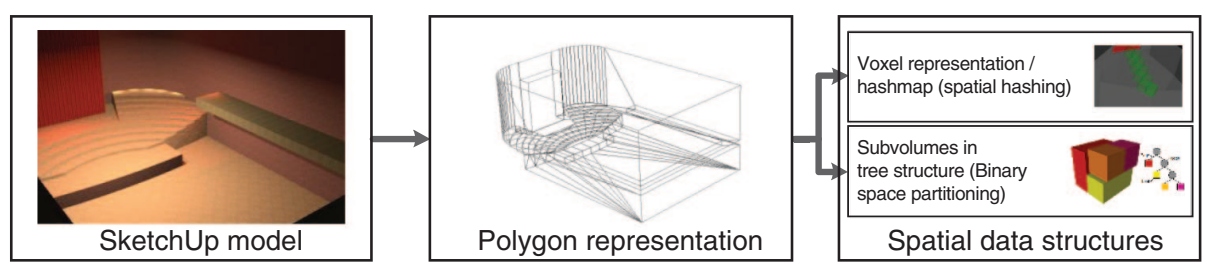

Figure 2. The room model is represented in two different spatial data structures at the same time. This allows for an optimal strategy to update the impulse response in real-time.

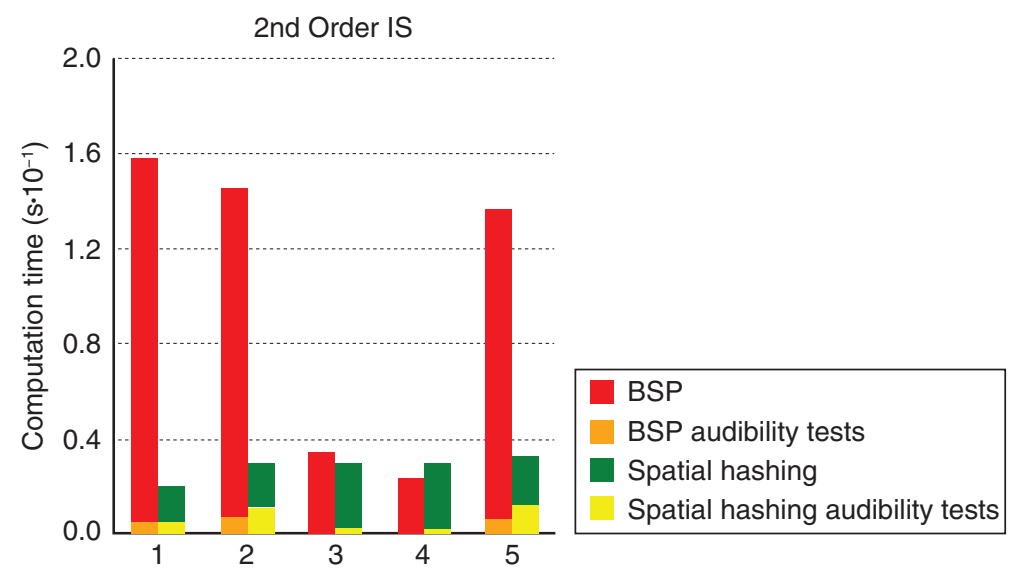

Figure 3. Example of a scene modification with update times for BSP and SH techniques for five different rooms. Although the audibility tests are slower using the SH structure, low order early reflections with limited intersections can quickly be computed due to the fast geometry update. (Fig. from Schröder ${ }^{9}$ )

up-to-date nearly in real-time and can be used to quickly update the psychoacoustically dominant direct sound and early reflections which need only a limited number of intersections. In five tested example rooms the calculation of two orders of image sources after scene modifications required between $10 \mathrm{~ms}$ to $30 \mathrm{~ms}$ computation time including the update of the hash-map and subsequent audibility tests. In contrast, the BSP-update with subsequent audibility tests required as much as $30 \mathrm{~ms}-160 \mathrm{~ms}$, as shown in Figure 3.

But this advantage is only preserved for a limited number of intersection tests. For the diffuse decay, where millions of intersections have to be tested, it is therefore always worthwhile to wait for the BSP update and then catch up with much faster intersections. 


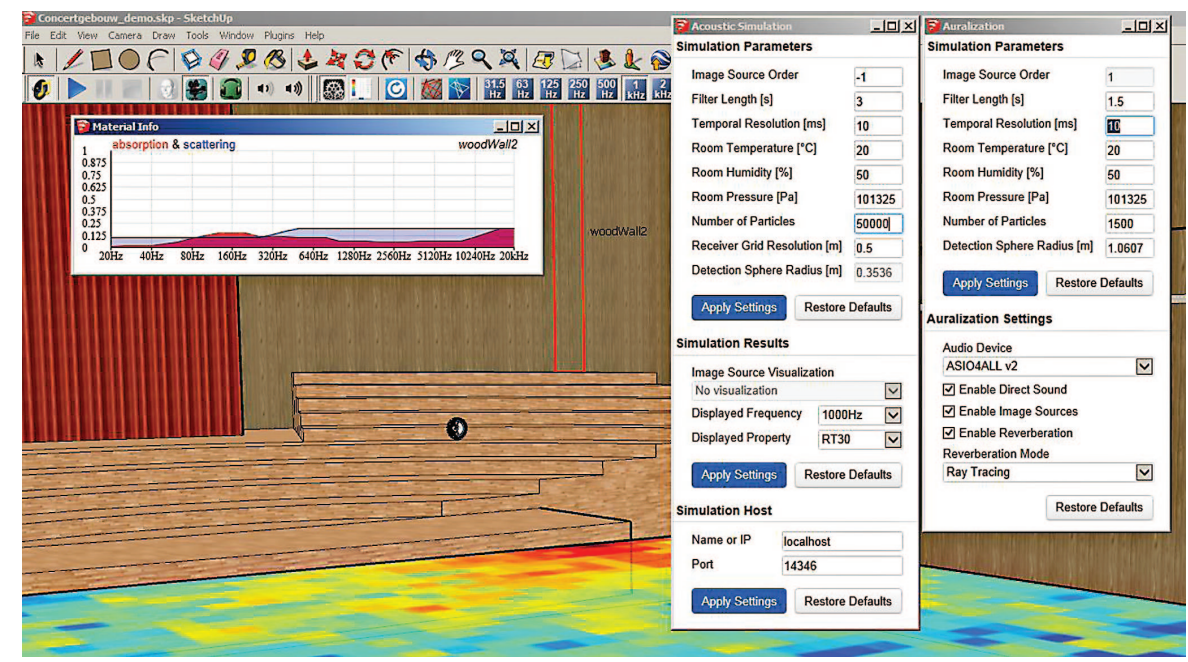

Figure 4. Screenshot of the graphical user interface, containing configuration dialogs for Acoustic Simulation (Visualization of room acoustic parameters) and Auralization.

\subsection{User interface}

The key requirement for the newly developed user interface was intuitive and easy-touse handling. This was achieved by integrating the acoustics functions seamlessly into the GUI of the CAD modeler SketchUp. Preset models for sound sources and receivers can be placed into the scene just as any other objects. Exchanging sound signals, directivities or the HRTF dataset can be done by just right-clicking the objects and extended context menus. An additional toolbar allows starting the real-time auralization, switching visualized frequencies and opening settings dialogues, as shown in Figure 4. Results of the current room situation are immediately audible on the headphones and also shown by coloured evaluation surfaces which can freely be placed inside the room.

\subsection{Framework}

Core of the simulation framework is the 3D modeling environment, in this case SketchUp. This software allows to be extended using plug-ins which have to be written in Ruby. The Ruby plug-in integrates a TCP/IP server which can connect to multiple clients. These clients can request a live update of the room geometry including continuous real-time updates. Two types of clients were implemented, both being able to run simulations on the geometry including image sources and ray tracing. The first client type is able to calculate room acoustics parameters (such as reverberation time, clarity, etc.) and returns a texture ready to be rendered inside SketchUp. The second 


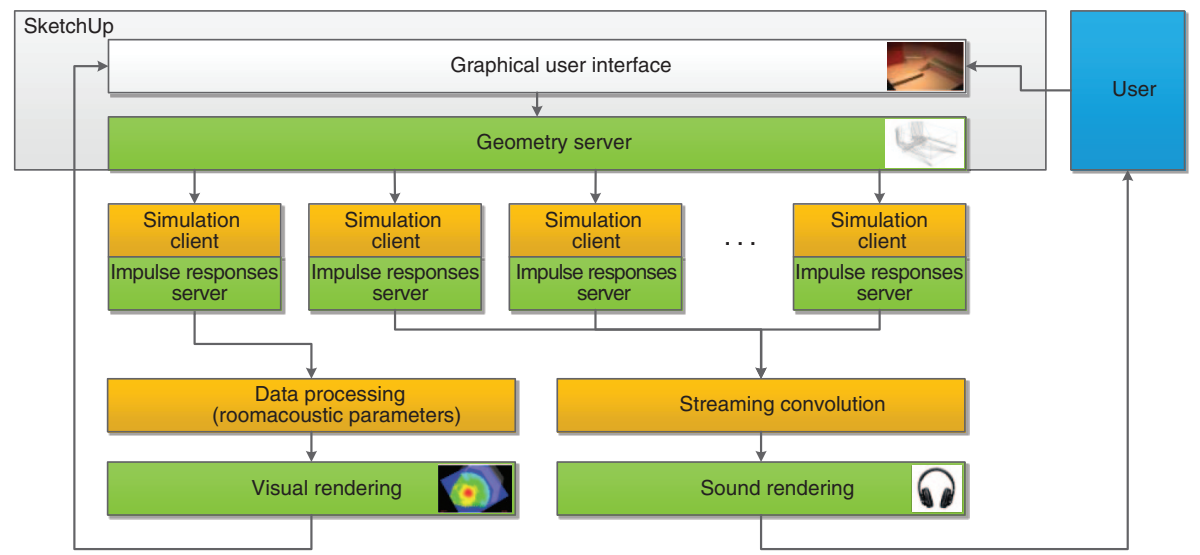

Figure 5. System framework. A Ruby plug-in for SketchUp streams geometry updates live over TCP/IP channels to multiple clients. The clients render room impulse responses which can either be evaluated to render room acoustic parameter surface plots back into SketchUp or to be directly injected into a low-latency streaming convolution engine for real-time auralization.

client renders room impulse responses directly into a low-latency streaming convolution engine, providing live feedback to the user, e.g. as a binaural stream. The general structure is shown in Figure 5.

\section{IMPLEMENTATION}

The concept behind this framework is to distribute the workload of complex simulations, e.g. with a multitude of sound sources, over multiple nodes. A switching matrix allows to assign certain sources and receivers to connected clients. The results can be mixed together to listen to a full orchestra, but they can also be handled separately, e.g. to render sound for individual receivers at separate positions.

For clients with multiple CPU cores, a multi-threading approach is followed that will run all simulation jobs in parallel. This includes the update of spatial data structures, the (image sources generation and audibility test, ray tracing and separate filter synthesis for each component. The parallel processing if these jobs is illustrated in Figure 6. If only one single machine is available, all components and jobs still can be run on this one PC, too.

It can be seen that in the example of Figure 6 the acoustically dominant direct sound component is up-to-date after only $25 \mathrm{~ms}$. This enables the simulation framework to be also used for interactive walkthroughs and moving sound sources in an immersive application. Also the use of a head-tracker becomes feasible. 


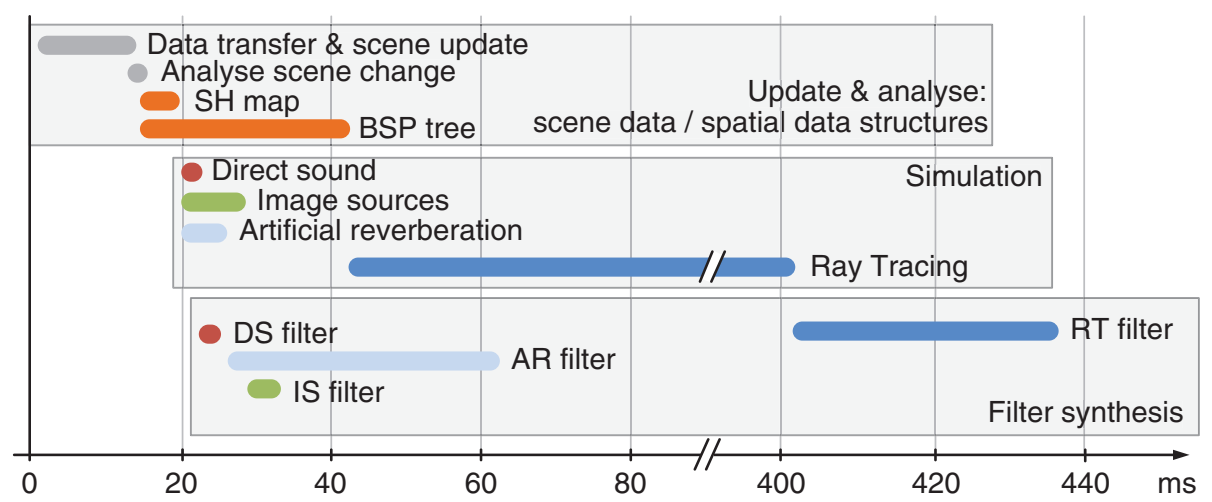

Figure 6. Parallel processing on a simulation node and exemplary calculation times after a geometry modification.

\section{APPLICATION}

With the features that are now available from inside the CAD modeling tool, it is possible for an architect to inspect the room acoustics already at early stages in the design process. This can avoid mistakes in a project phase where major changes are more likely to be done. Acoustic consultants can speed up their workflow by immediately evaluate treatments to the room acoustics. Also it is comfortable to present the proposed corrections to the client using the possibilities of quick changes and virtual walkthroughs. For the same reason it can be recommended to use the plug-in in teaching and education for acousticians and architects due to the strength in demonstrations. It is even thinkable that sound engineers can make use of this room acoustic simulation for sound design purposes, as an alternative to the popular convolution reverberation plug-ins, which usually use measured impulse responses and are thus limited to a certain selection of rooms.

\section{CONCLUSION}

A fully operable room acoustics simulation software including the state-of-the-art simulation models was implemented into a popular 3-D modeling tool, as used by many architects. The seamless integration and reduced set of controls enables non-expert users to run acoustic simulations and also perform auralizations, which provide immediate feedback of the currently designed scene. The simulation reacts in real-time to any changes of the scene or configuration of sources and receivers, so that professional and non-expert users can quickly evaluate or hear the consequences of their ideas.

Due to a highly parallelized threading concept with prioritization on fast updates of psychoacoustically dominant components, the simulation provides an immersive rendering of the surrounding scene and can be used in interactive experiments. A future version will therefore include head-tracking. 
Also the balancing of simulation workload which is currently done manually for multiple simulation nodes will be replaced by an automatic job manager for application on bigger clusters, such as the aixCAVE.

The modular concept of the simulation framework is prepared for different sound renderers, including Ambisonics, Vector-base Amplitude Panning, binaural synthesis, headtracked binaural synthesis, Crosstalk-Cancellation and stereo.

\section{ACKNOWLEDGMENTS}

We gratefully thank the German Research Foundation (DFG) for partly funding internals behind this project.

\section{REFERENCES}

[1] G. Naylor, "ODEON - Another Hybrid Room Acoustical Model" Applied Acoustics, vol. 38, pp. 131-143 (1993).

[2] M. Vorländer, "International Round-robin on Room Acoustical Computer Simulation", in Proceedings of 15th ICA, pp. 689-692, (Trondheim, 1995).

[3] I. Bork, "A comparison of room simulation software - the 2nd round robin on room acoustical computer simulation" in Acta Acustica united with Acustica, vol. 86, pp. 943-956 (2000).

[4] I. Bork, "Report on the 3rd round robin on room acoustical computer simulation - Part I: Measurements" in Acta Acustica united with Acustica, vol. 91, pp. 740-752 (2005).

[5] I. Bork, "Report on the 3rd round robin on room acoustical computer simulation - Part II: Calculations", in Acta Acustica united with Acustica, vol. 91, pp. 753-763 (2005).

[6] L. Savioja et al., "Creating Interactive Virtual Acoustic Environments," J. Audio Engineering Society, vol. 47, no. 9, pp. 675-705 (1999).

[7] A. Chandak et al., "Ad-frustum: Adaptive frustum tracing for interactive sound propagation", IEEE Transactions on Visualization and Computer Graphics, vol. 14 (2008).

[8] B.-I. Dalenbäck and M. Strömberg, "Real time walkthrough auralization - the first year" in Proceedings IOA Copenhagen, 2006.

[9] D. Schröder, "Physically based real-time auralization of interactive virtual environments", $\mathrm{PhD}$ Thesis, Fakultät für Elektrotechnik und Informationstechnik der Rheinisch-Westfälischen Technischen Hochschule Aachen, 2012.

[10] M. R. Schroeder, "Frequency-correlation functions of frequency responses in rooms" in The Journal of the Acoustical Society of America, vol. 34, no. 12, pp. 1819-1823 (1962)

[11] J. Allen and D. Berkley, "Image method for efficiently computing small-room acoustics", Journal of the Acoustical Society of America, vol. 65, pp. 934-950 (1979).

[12] A. Krokstad, S. Strom, and S. Sorsdal. Calculating the acoustical room response by the use of aray tracing technique. Journal of Sound and Vibration, 8(1):118-125, 1968 . 
